\title{
Risk Factor Profile, Clinical and Vascular Territory Involved in Patients of Stroke Presenting to a Tertiary Care Hospital in India Over 1 Year
}

\author{
Amit Sreen ${ }^{1}$, Prafull Sharma ${ }^{2}$, Vivek Guleria ${ }^{3}$, Niket Verma ${ }^{4}$
}

\begin{abstract}
Introduction: Recent rapid socioeconomic changes have led to a concomitant change in people's lifestyle, leading to work-related stress and altered food habits which leads to enhanced atherosclerosis and strokes.

Aim: To analyze the risk factor profile, clinical, and vascular territory involved in all patients of stroke presenting to tertiary care hospital over 1 year. Materials and methods: All cases of fresh stroke reporting, referred, or transferred to this hospital (within 2 weeks of ictus) who were more than 15 years of age were included. A detailed history was obtained regarding time and mode of onset of symptoms and risk factor details following clinical evaluation, patients underwent complete hemogram, blood sugar levels (fasting and post prandial), lipid profile, and other metabolic parameters. All patients were subjected to chest radiography 12-lead electrocardiography (ECG) and two-dimensional (2D) echocardiography to detect cardiac abnormalities. Neuroimaging was performed in all in the form of noncontrast computed tomography (CT) head and magnetic resonance imaging (MRI) brain, while magnetic resonance angiography (MRA) brain was carried out in selected cases.

Results: Ischemic strokes were higher (77.4\%) when compared with hemorrhagic strokes (22.6\%). The maximum number of patients was in the age group of 51-60 years (37.15\%). Males were more affected than females, both in ischemic as well as hemorrhagic stroke. The most common risk factor was hypertension (65.35\%), followed by smoking $(46.26 \%)$ and diabetes $(21.05 \%)$. Alcohol usage was more significant in cases of hemorrhagic stroke (11.14\%). More number of patients reported stroke symptoms on awakening in ischemic stroke. Anterior vascular territory was more involved (76.8\%). Left hemiparesis was more common (62.05\%), and aphasia was seen in $27.97 \%$ of patients. Middle cerebral artery (MCA) territory was most commonly involved (78\%), and gangliocapsular region was most commonly affected (41.54\%) Lacunar infarcts were seen in $22 \%$ cases. In hemorrhagic stroke, the basal ganglia was most commonly involved ( $56.17 \%)$, followed by thalamus ( $26.03 \%)$.

Conclusion: Developing countries like India are facing burden of lifestyle diseases. Stroke is leading cause of death and disability in India.

Keywords: Brain ischemia, Risk factors, Stroke.

Journal of Medical Academics (2019): 10.5005/jp-journals-10070-0037
\end{abstract}

\section{INTRODUCTION}

The World Health Organization defines the stroke in an adult as "rapidly developing clinical symptoms and signs of focal (at times global) disturbance of cerebral function, lasting for more than 24 hours or leading to death with no apparent cause other than that of vascular origin." ${ }^{11}$ Globally, stroke is the third commonest cause of mortality and the fourth leading cause of disease burden.

The prevalence rate of stroke in India is $545 / 100,000 .^{2}$ Recent studies showed that the age-adjusted annual incidence rate in India is $105 / 100,000$ in the urban community and 262/100,000 in a rural community. 3,4

In terms of population, India ranks second only to China. Recent rapid socioeconomic changes have led to a concomitant change in people's lifestyle, leading to work-related stress and altered food habits, raising the risk of hypertension. These factors, coupled with an increase in the average life expectancy, are expected to have an impact on the occurrence of stroke disorder in India.

This study aims to analyze the risk factor profile, clinical, and vascular territory involved in all patients of stroke presenting to tertiary care hospital over 1 year.

\section{Materials and Methods}

The study was carried out in Army Hospital (Research and Referral), which is a tertiary care hospital for the armed forces between December 2018 and December 2019. All cases of fresh stroke
${ }^{1}$ Department of Neurology, Army Hospital Research and Referral, New Delhi, India

${ }^{2}$ Department of Medicine and Cardiology, Army Hospital Research and Referral, New Delhi, India

${ }^{3}$ Department of Cardiology, Army Hospital Research and Referral, New Delhi, India

${ }^{4}$ Department of General Medicine, Army College of Medical Sciences, New Delhi, India

Corresponding Author: Prafull Sharma, Department of Medicine and Cardiology, Army Hospital Research and Referral, New Delhi, India, Phone: +91 9971829207, e-mail: drprafullsharma@gmail.com

How to cite this article: Sreen A, Sharma P, Guleria V, et al. Risk Factor Profile, Clinical and Vascular Territory Involved in Patients of Stroke Presenting to a Tertiary Care Hospital in India Over 1 Year. J Med Acad 2019;2(2):54-57.

Source of support: Nil

Conflict of interest: None

reporting, referred, or transferred to this hospital (within 2 weeks of ictus) who were more than 15 years of age were included, while follow-up cases of stroke (stroke onset beyond 2 weeks) and patients in whom stroke could not be confirmed by neuroimaging were excluded in this study. This observational study was carried out between December 2018 and December 2019 after approval from the ethical committee.

(C) The Author(s). 2019 Open Access This article is distributed under the terms of the Creative Commons Attribution 4.0 International License (https://creativecommons. org/licenses/by-nc/4.0/), which permits unrestricted use, distribution, and non-commercial reproduction in any medium, provided you give appropriate credit to the original author(s) and the source, provide a link to the Creative Commons license, and indicate if changes were made. The Creative Commons Public Domain Dedication waiver (http://creativecommons.org/publicdomain/zero/1.0/) applies to the data made available in this article, unless otherwise stated. 


\section{Data Collection Technique and Tools}

Patients were included in the study after an informed consent from them or their immediate relatives. A detailed history was obtained from the patient or relatives with emphasis on time of onset of symptoms, mode of onset of symptoms, and risk factor details. Following clinical evaluation, patients underwent the following investigations: complete hemogram, blood sugar levels (fasting and post prandial), lipid profile (12-hour fasting state), and other metabolic parameters. All patients were subjected to chest radiography (for any evidence of cardiomegaly), 12-lead ECG, and 2D echocardiography to detect cardiac abnormalities. Neuroimaging was performed in all in the form of noncontrast CT head and MRI brain (T1, T2, flair, and diffusion studies), while MRA brain was carried out in selected cases.

Data were analyzed in a descriptive manner, and $p$ values were calculated using the Chi-square test.

\section{Results}

A total of 339 patients were screened, out of which 323 patients were finally included in the study. Out of 16 patients who were excluded, 11 were lost to follow-up and 5 patients did not turn out to have a stroke. There were 190 males and 133 females.

\section{Stroke Type}

Ischemic strokes were higher in number (total of 250 patients; $77.4 \%$ ) when compared with intracranial hemorrhage (ICH) (total of 73 patients; $22.6 \%$ ). This distribution of stroke patients between ischemic and ICH was found to be statistically significant $(p<0.001)$.

\section{Age Distribution}

Majority of the patients were in the age group of 51-60 years (37.15\%), closely followed by $41-50$ years age group ( $24.46 \%)$. The distribution of patients in the given age groups was found to be statistically significant ( $p<0.001$; Table 1$)$.

In $\mathrm{ICH}$, more patients were above 60 years of age $(52.04 \%$; Table 2). The age distribution as per type of stroke was found to be significant in all age groups $(p<0.001)$ except patients greater than 70 years $(p=0.157)$.

\section{Social Background}

Rural patients had a higher incidence (59.75\%) when compared with the urban group (40.25\%). The distribution of patients based on their social background was not found to be statistically significant $(p=0.136)$.

\section{Sex Distribution}

Males were more in both the groups (ischemic - 56\%; ICH-68.5\%), although the difference between the two sexes was more

Table 1: Age distribution

\begin{tabular}{llcc}
\hline & & $\begin{array}{c}\text { Ischemic stroke } \\
(n=250)(\%)\end{array}$ & $\begin{array}{l}\text { Hemorrhagic stroke } \\
(n=73)(\%)\end{array}$ \\
\hline Age (years) & $15-40$ & $9(23)$ & $9.5(7)$ \\
& $41-50$ & $5(13)$ & $17.8(13)$ \\
& $51-60$ & $6(15)$ & $20.5(15)$ \\
& $61-70$ & $11(28)$ & $38.3(28)$ \\
Sex & $>70$ & $4(10)$ & $13.7(10)$ \\
& Male & $56(140)$ & $68.5(50)$ \\
& Female & $44(110)$ & $31.5(23)$ \\
\hline
\end{tabular}

Table 2: Regional distribution of infarcts $(n=250)$

\begin{tabular}{rlc}
\hline & Region & $\begin{array}{c}\text { Number of } \\
\text { patients (\%) }\end{array}$ \\
\hline 1 & Cortex (frontal, parietal, and lateral temporal) & $73(29)$ \\
2 & Occipital cortex & $15(6)$ \\
3 & Medial temporal cortex & $4(1.6)$ \\
4 & Subcortical white matter & $56(22.4)$ \\
5 & Gangliocapsular & $65(26)$ \\
6 & Thalamus & $13(5.2)$ \\
7 & Midbrain & $7(2.8)$ \\
8 & Pons & $5(2)$ \\
9 & Medulla & $5(2)$ \\
10 & Cerebellum & $7(2.8)$ \\
\hline
\end{tabular}

prominent in the ICH group. However, this difference was not found to be statistically significant ( $p=0.056)$.

\section{Risk Factors}

The prevalence of risk factors in the study group was as followshypertension $65.35 \%(n=211)$, diabetes mellitus (DM) $21.05 \%$ ( $n=68)$, smoking $46.26 \%(n=149)$, and significant alcohol use $11.14 \%$ ( $n=36$ ). Significant alcohol use was defined as alcohol consumption of more than $80 \mathrm{~g}$. Hypertension was found to be the most common risk factor.

\section{Echocardiography}

A total of 293 patients (90.71\%) underwent echocardiography. In total, $27.30 \%$ patients had left ventricular hypertrophy, with normal ejection traction, and $56.99 \%$ had a normal study.

Out of 250 patients having ischemic stroke, 138 patients (55.2\%) reported weakness on awakening in the morning when compared with 112 patients (44.8\%), who developed acute onset weakness during daily activities. The mode of onset of stroke was assessed only in 112 patients, who had stroke while being awake. Aphasia was observed in 75 patients (29.97\%). Motor aphasia was most commonly present ( $n=22 ; 8.98 \%)$ followed by global aphasia $(n=$ $20 ; 7.78 \%)$. Dysarthria was more common in anterior circulation ischemic strokes (38.4\%) when compared with posterior circulation (5.6\%) and was observed more in patients with left hemiparesis (33.2\%). A total of 120 patients (48\%) had sensory symptoms. Unilateral hemisensory loss was present in 111 patients, while crossed involvement was seen in 3 patients. Majority of patients (76.8\%) had involvement of anterior territory when compared with posterior territory involvement (23.2\%; Table 2). A total of 121 patients $(62.05 \%)$ had left-sided involvement compared with 74 patients (37.95\%) who showed right-sided involvement. Facial paresis was observed in 206 patients (82.4\%). Right upper motor neuron paresis was found in 92 patients (36.8\%) and left-sided involvement in 114 patients (45.6\%). Seizures were present in 22 patients (9\%), while 27 patients $(10.8 \%)$ had a history of transient ischaemic attack (TIA) in the past.

In ICH patients, 41 patients (56.17\%) had basal ganglia involvement, 10 patients (13.69\%) had lobar bleeds, 19 patients $(26.03 \%)$ had thalamic involvement, while cerebellar involvement was seen in 3 patients (4.10\%; Table 3 ). Hypertension was seen in 49 patients (67.1\%) and oral anticoagulant use was present in 5 patients (6.84\%). Headache was present in 21 patients (28.76\%), vomiting in 18 patients $(24.66 \%)$, while altered sensorium at presentation was seen in 20 patients (27.38\%) and speech disturbance in $14 \%$ patients. 
Risk Factor Profile, Clinical and Vascular Territory Involved in Patients of Stroke

Table 3: Location of intracranial hemorrhage

\begin{tabular}{llc}
\hline & Location & $\begin{array}{l}\text { Number of patients } \\
(n=73)(\%)\end{array}$ \\
\hline 1 & Basal ganglia & $56.16(41)$ \\
2 & Lobar & $13.7(10)$ \\
3 & Cerebellum & $4.12(3)$ \\
4 & Thalamus & $26.02(19)$ \\
\hline
\end{tabular}

\section{Discussion}

This study was carried out at the Department of Neurology, Army Hospital (Research and Referral) — a tertiary care center. In this study, ischemic strokes were higher (77.4\%) than ICH (22.6\%), consistent with the findings of several past studies on stroke. ${ }^{5}$ However, in the west, the incidence of ICH has been found to be slightly higher. ${ }^{6}$ The maximum number of patients was in the age group of 51 to 60 years (37.15\%). In total, $24.45 \%$ patients were in the age group of 41 to 50 years and $24.15 \%$ in the age group of $61-70$ years. According to the Lausanne Stroke Registry (1988) of 1,000 patients, most patients were in the age group of $60-80$ years. The same stroke registry documented that the mean age was $60.7+13.9$ years for the entire group. ${ }^{7}$ Our study showed a slightly lower age group which was most likely due to the fact that stroke in Indian setup occurs at least a decade earlier than the west and elderly patients may have gone unreported in our setup.

Like other studies, this study also showed a general male preponderance in stroke prevalence. ${ }^{7-9}$ Hypertension is one of the most important modifiable risk factors. It predisposes to ischemic as well as hemorrhagic strokes by aggravating atherosclerosis. Hypertension increases the relative risk for stroke by three to four times. Our study showed a presence of hypertension in $65.35 \%$ of patients, and around $37 \%$ of these patients had hypertension of more than 5 years duration. Kumral and Bayulkem ${ }^{10}$ reported hypertension in $62 \%$ of patients with ischemic stroke, while Arboix et al. ${ }^{11}$ reported an incidence of $61.7 \%$ in $\mathrm{ICH}$ patients.

Studies have reported the relative risk of stroke of all ages to be 1.8 for diabetic men and 3.0 for diabetic women. ${ }^{12}$ In the Lausanne Registry, ${ }^{7}$ DM was seen in $12.6 \%$ of patients, while the Barcelona Stroke Registry documented $21 \%$ of patients with diabetes among the ischemic strokes. ${ }^{8}$ In ischemic patients, diabetes has been reported in $26 \%$ cases, and in hypertensive bleed patients, diabetes has been reported in $17-30 \%$ cases. $^{10}$

Consistent with the above studies, we found DM in $21.05 \%$ of patients. In total, $3 \%$ of these patients were diagnosed to have DM during this present admission, after having ruled out the possibility of stress-induced hyperglycemia with the help of $\mathrm{HbA1C}$ and follow-up blood glucose testing after 1 week to 10 days. In our study, $46.26 \%$ patients were found to be smokers. Previous studies have shown a similar prevalence of smoking among stroke patients. The prevalence of smoking was found to be $45.6 \%$ in the Lausanne Stroke Registry. ${ }^{7}$ Kumral and Bayulkem ${ }^{10}$ reported smoking in $28 \%$ of the patients. Alcohol consumption may increases the risk of stroke through changes in blood pressure, platelet aggregability, blood coagulation, and the level of triglycerides (TGLs). In this study, 36 patients had a history of significant alcohol use among the total 323 patients (11.14\%). Significant alcohol use has been reported to be in $12.8 \%$ of ICH patients. ${ }^{13}$ In total, $62.85 \%$ patients in our study had low-density lipoprotein value of more than $160 \mathrm{mg} / \mathrm{dL}$, $85.75 \%$ of patients had TGL of more than $150 \mathrm{mg} / \mathrm{dL}$, and $60.06 \%$ of patients had high-density lipoprotein value of less than $40 \mathrm{mg} /$ dL. Abreu et al. ${ }^{14}$ reported hyperlipidemia in $70 \%$ of patients, while the Lausanne Stroke Registry ${ }^{7}$ reported an incidence of $14.5 \%$. The more number of patients with hyperlipidemia in our study could be due to increase in the prevalence of metabolic syndrome and the changing lifestyle among the Indian population.

In our study, 52.2\% of the total patients reported features of stroke on awakening, while $44.8 \%$ suffered from stroke during daily activities. In this study, the mode of onset was assessed only in those patients who had stroke while they were awake. Of the 112 patients, stroke onset was sudden and acute in 86 patients (76.78\%), stepwise and gradual progression in 22 patients (19.64\%) followed by fluctuating course in 4 patients $(3.58 \%)$. The mode of onset of stroke could not be defined clearly in the remaining patients who noticed stroke immediately on awakening. In the Harvard Cooperative Stroke Registry, deficit was sudden at onset in 53\%, stepwise or gradual in 38\%, and fluctuating in 9\% of patients. ${ }^{15}$

In our study, aphasia was found in 75 patients (29.97\%). Broca's aphasia was the most common type seen in $8.98 \%$, followed by global aphasia in $7.78 \%$, and Wernicke's aphasia in $5.92 \%$ patients. Dysarthria was documented in $44 \%$ of patients. In the Lausanne Stroke Registry, ${ }^{7}$ aphasia was seen in $33.6 \%$ of patients. The commonest type was motor aphasia in $12.7 \%$, followed by global aphasia in $11.2 \%$ and sensory aphasia in $9.7 \%$. Dysarthria was documented in $12.4 \%$ of patients. In our study, sensory disturbances were found in 120 patients (48\%). Left side was involved in $25.60 \%$, right side in $21.20 \%$, and crossed sensory deficit was seen in $1.2 \%$ patients. The Lausanne Stroke Registry ${ }^{7}$ reported sensory deficit in $46.4 \%$ cases, and the Barcelona Stroke Registry ${ }^{8}$ reported sensory disturbances in $47 \%$ cases.

Our study showed that carotid territory (anterior circulation) was affected in $82.4 \%$ of patients, vertebrobasilar territory (posterior circulation) was involved in $16 \%$ of patients, and both were affected in $1.6 \%$ of patients. The Barcelona Stroke Registry (1998) reported that anterior circulation was affected in $70 \%$ of patients and posterior circulation in $12 \%$ of patients. ${ }^{8}$

In this study, MCA was affected in $78 \%$ of total patients, followed by posterior cerebral artery (PCA) in $8.8 \%$, anterior cerebral artery (ACA) in $5.2 \%$, and posterior inferior cerebellar artery in $3.2 \%$ of patients. Basilar artery was involved in $2.4 \%$, superior cerebellar artery in $0.8 \%$, and anterior inferior cerebellar artery in $0.41 \%$ of the total patients. Two patients had both MCA and ACA infarcts, and one patient had both MCA and PCA infarcts. Caplan et al. ${ }^{15}$ documented that MCA was involved in $75 \%$ followed by PCA in $11 \%$, ACA in $3 \%$, and basilar artery in $5 \%$ of cases.

In our study, motor deficit in the form of hemiparesis was the commonest presentation and was seen in 195 patients, constituting $78 \%$ of the total. Left hemiparesis was more common (62.05\%) than right hemiparesis (37.95\%).

The results of our study are similar to the reports of the Lausanne Stroke Registry ${ }^{7}$ in which $83.2 \%$ of patients had a motor deficit. However, Bogousslavsky et al. reported right hemiparesis to be more common (56\%) than left hemiparesis.

In this study, $91.5 \%$ of hemiparesis was due to anterior circulation stroke and $8.5 \%$ was due to posterior circulation stroke, which in addition to hemiparesis had other manifestations. The upper and lower limbs were found to be equally involved in $68.2 \%$ of patients, upper limb more involved in $17.95 \%$, and lower limb more involved in $13.84 \%$ patients. All the 13 ACA infarcts showed more lower limb (LL) involvement and 5 patients with MCA infarcts 
also manifested with predominant LL involvement. Faciobrachial monoparesis was seen in 14 patients (2.5\%).

In this study, $29.2 \%$ of total infarcts involved frontal, parietal, and lateral temporal cortex in various combinations. This was followed by gangliocapsular region involvement in $26 \%$ (internal capsule: $19.6 \%$, basal ganglia: $6.32 \%$ ) and subcortical white matter in $22.4 \%$, which involved frontal, parietal, and temporal white matter. Among vertebrobasilar artery infarcts, the medial occipital cortex was most commonly involved (6.7\%), followed by cerebellum (2.8\%) and thalamus (5.2\%). Midbrain (2.8\%), medulla (2\%), and pons (2\%) were the other regions involved.

In the Barcelona Stroke Registry, the main locations, either isolated or in combination, of cerebral infarcts were parietal (33.6\%), temporal (29\%), frontal (29\%), internal capsule (18.5\%), occipital cortex (9\%), basal ganglia (7\%), thalamus (5.5\%), pons (9\%), medulla (4.5\%), and the midbrain (3.5\%). ${ }^{8}$

Our study showed basal ganglia bleed to be the most common site $(56.17 \%)$ followed by thalamic bleeds (26.03\%), lobar bleeds (13.69\%), and cerebellar involvement (4.10\%). Prevalence of thalamic bleeds have been variedly reported in a previous stroke studies with one study reporting a prevalence of $6 \%{ }^{16}$

Hypertension was found to be the most common cause of $\mathrm{ICH}$ at $67.1 \%$ of total bleed cases. Oral anticoagulants were found to be responsible in $6.84 \%$ of cases.

\section{Conclusion}

In India, the incidence of stroke is increasing nowadays. There is a huge burden of stroke with significant regional variations of stroke in our county. Ischemic strokes are higher (77.4\%) when compared with hemorrhagic strokes (22.6\%). The maximum number of patients was in the age group of $51-60$ years (37.15\%). Males were more affected than females, both in ischemic and hemorrhagic strokes. The most common risk factor was hypertension (65.35\%), followed by smoking (46.26\%) and diabetes (21.05\%). Alcohol usage was more significant in cases of hemorrhagic stroke (11.14\%). More number of patients reported stroke symptoms on awakening in ischemic stroke. Anterior vascular territory was more involved (76.8\%). Left hemiparesis was more common (62.05\%), and aphasia was seen in $27.97 \%$ of patients. Middle cerebral artery territory was most commonly involved (78\%), and gangliocapsular region was most commonly affected (41.54\%). Lacunar infarcts were seen in $22 \%$ cases. In hemorrhagic stroke, the basal ganglia was most commonly involved (56.17\%), followed by thalamus $(26.03 \%)$. Headache (28.76\%) and loss of consciousness (27.39\%) were the most commonly associated features of hemorrhagic stroke.

\section{References}

1. World Health Organization. International Task Force for Prevention of Coronary Heart Disease and Stroke, Etiology and Epidemiology of Stroke. WHO, Geneva: WHO 1980.

2. Das SK, Banerjee TK, Biswas A, et al. A prospective community-based study of stroke in Kolkata, india. Stroke 2007;38(3):906-910. DOI: 10.1161/01.STR.0000258111.00319.58.

3. Bhattacharya S, Saha SP, Basu A, et al. A 5-year prospective study of incidence, morbidity and mortality profile of stroke in a rural community of eastern india. J Indian Med Assoc 2005;103(12):655659.

4. Banerjee TK, Mukherjee CS, Sarkhel A. Stroke in the urban population of Calcutta - an epidemiological study. Neuroepidemiology 2001;20(3):201-207. DOI: 10.1159/000054788.

5. Lavados PM, Sacks C, Prina L, et al. Incidence, 30-day case-fatality rates, and prognosis of stroke in lquique, chile: a 2-year community based prospective study (PISCIS project). Lancet. 2005;365(9478):2206-2215.

6. Das K, Banerjee TK. Stroke: Indian scenario. Circulation 2008;118(25):2719-2724. DOI: 10.1161/CIRCULATIONAHA.107. 743237.

7. Bogousslavsky J, Van Melle G, Regli F. The Lausanne stroke registry: Analysis of 1,000 consecutive patients with first stroke. Stroke 1988;19:1083-1092. DOI: 10.1161/01.STR.19.9.1083.

8. Marti-Vilalta JL, Arboix A. The Barcelona stroke registry. Eur Neurol 1999;41(3):135-142. DOI: 10.1159/000008036.

9. MacMahon S, Rodgers A. Primary and secondary prevention of stroke. Clin Exp Hypertens 1996;18(3-4):537-546. DOI: 10.3109/10641969609088983.

10. Kumral E, Bayulkem G. Spectrum of single and multiple corona Radiata infarcts: clinical/MRI correlations. J Stroke Cerebrovasc Dis 2003;12:66-73. DOI: 10.1053/jscd.2003.11.

11. Arboix A, Rodríguez-Aguilar $R$, Oliveres $M$, et al. Thalamic haemorrhage vs internal capsule-basal ganglia haemorrhage: clinical profile and predictors of in-hospital mortality. BMC Neurol 2007;7:32. DOI: 10.1186/1471-2377-7-32.

12. Burchfield CM, Curb JD, Rodriguz BL, et al. Glucose intolerance and 22-year stroke incidence: the Honolulu heart program. Stroke 1994;25(5):951-957. DOI: 10.1161/01.STR.25.5.951.

13. Gill JS, Shipley MJ, Tsementzis SA, et al. Alcohol consumption - a risk factor for hemorrhagic and non-hemorrhagic stroke. Am J Med 1991;90(4):489-497. DOI: 10.1016/0002-9343(91)80090-9.

14. Abreu TT, Mateus S, Correia J. Therapy implications of transthoracic echocardiography in acute ischemic stroke patients. Stroke 2005;36:1565-1566. DOI: 10.1161/01.STR.0000170636.08554.49.

15. Mohr JP, Caplan LR, Melski JW, et al. The Harvard cooperative stroke registry: A prospective registry. Neurology 1978;28(8):754-762. DOI: 10.1212/WNL.28.8.754.

16. Gross CR, Kase CS, Mohr JP, et al. Stroke in South Alabama: incidence and diagnostic features - a population based study. Stroke 1984;15(2):249-255. DOI: 10.1161/01.STR.15.2.249. 